\title{
The Shape of Production Functions and the Direction of Technical Change
}

\author{
Preliminary - Comments appreciated \\ Charles I. Jones* \\ Department of Economics, U.C. Berkeley and NBER \\ E-mail: chad@econ.berkeley.edu \\ http://elsa.berkeley.edu/ chad
}

March 19, 2004 - Version 0.5

\begin{abstract}
This paper views the standard production function in macroeconomics as a reduced form and derives its properties from microfoundations. The shape of this production function is governed by the distribution of ideas. If that distribution is Pareto, then two results obtain: the global production function is Cobb-Douglas, and technical change in the long run is labor-augmenting. Kortum (1997) showed that Pareto distributions were necessary if search-based idea models were to exhibit steady-state growth. Here we show that this same assumption delivers the additional results about the shape of the production function and the direction of technical change.
\end{abstract}

Key Words: Cobb-Douglas, Pareto, Power Law, Labor-Augmenting Technical Change, Steady State Growth

JEL Classification: O40, E10

* I am grateful to Susanto Basu, Francesco Caselli, Hal Cole, Xavier Gabaix, Doug Gollin, Pete Klenow, Robert Solow, Alwyn Young, and seminar participants at Arizona State University, the Chicago GSB, the San Francisco Fed, Stanford, U.C. Berkeley, UCLA, U.C. Davis, and U.C. Santa Cruz for comments. Sam Kortum provided especially useful insights, for which I am most appreciative. Dean Scrimgeour supplied excellent research assistance. This research is supported by NSF grant SES-0242000. 


\section{INTRODUCTION}

Where do production functions come from? To take a common example, our models frequently specify a relation $y=f(k, \cdot)$ that determines how much output per worker $y$ can be produced with any quantity of capital per worker $k$. We typically assume the economy is endowed with this function, but what microfoundations can be provided to tell us more about it?

Suppose production techniques are discovered, i.e. they are ideas. One example of such an idea would be a Leontief technology that says, "for each unit of labor, take $k^{*}$ units of capital. Follow these instructions [omitted], and you will get out $y^{*}$ units of output." The values $k^{*}$ and $y^{*}$ are parameters of this production technique.

If one wants to produce with a very different capital-labor ratio from $k^{*}$, the Leontief technique is not particularly helpful, and one needs to discover a new idea "appropriate" to the higher capital-labor ratio. ${ }^{1}$ Notice that one can replace the Leontief structure with a production technology that exhibits a low elasticity of substitution, and this statement remains true: to take advantage of a substantially higher capital-labor ratio, one really needs a new technique targetted at that capital-labor ratio. One needs a new idea.

According to this view, the standard production function that we write down, mapping the entire range of capital-labor ratios into outputs, is a reduced form. It is not a single technology, but rather represents the substitution possibilities across different production techniques. The elasticity of substitution for this global production function depends on the extent to which new techniques that are appropriate at higher capital-labor ratios have been discovered. That is, it depends on the distribution of ideas.

But from what distribution are ideas drawn? Kortum (1997) examined a search model of growth in which ideas are productivity levels that are drawn from a distribution. He showed that the only way to get exponential

\footnotetext{
${ }^{1}$ This use of appropriate technologies is related to Basu and Weil (1998).
} 
growth in such a model is if ideas are drawn from a Pareto distribution, at least in the upper tail.

This same basic assumption, that ideas are drawn from a Pareto distribution, yields two additional results in the framework considered here. First, the global production function is Cobb-Douglas. Second, technological change is purely labor-augmenting in the long run. In other words, an assumption Kortum (1997) suggests we make if we want a model to exhibit steady-state growth leads to important predictions about the shape of production functions and the direction of technical change.

Section 2 of this paper presents a simple baseline model that illustrates all of the main results of this paper. In particular, that section shows how a specific shape for the technology frontier produces a Cobb-Douglas production function and labor-augmenting technical change. Section 3 develops the full model with richer microfoundations and derives the Cobb-Douglas result, while Section 4 discusses the underlying assumptions and the relationship between this model and Houthakker (1955-1956). Section 5 develops the implications for the direction of technical change. Section 6 provides a numerical example of the model, and Section 7 concludes.

\section{A BASELINE MODEL}

\subsection{Preliminaries}

Let a particular production technique — call it technique $i$ — be defined by two parameters, $a_{i}$ and $b_{i}$. With this technique, output $Y$ can be produced with capital $K$ and labor $L$ according to a (local) production function

$$
Y=\tilde{F}\left(b_{i} K, a_{i} L\right)
$$

We assume that $\tilde{F}(\cdot, \cdot)$ exhibits a low elasticity of substitution between its inputs and constant returns to scale in $K$ and $L$. In addition, we make the 
usual neoclassical assumption that $\tilde{F}$ possesses positive but diminishing marginal products and satisfies the Inada conditions.

This production function can be rearranged to give

$$
Y=a_{i} L \tilde{F}\left(\frac{b_{i} K}{a_{i} L}, 1\right),
$$

so that in per worker terms we have

$$
y=a_{i} \tilde{F}\left(\frac{b_{i}}{a_{i}} k, 1\right),
$$

where $y \equiv Y / L$ and $k \equiv K / L$. Now, define $y_{i} \equiv a_{i}$ and $k_{i} \equiv a_{i} / b_{i}$. Then the production technique can be written as

$$
y=y_{i} \tilde{F}\left(\frac{k}{k_{i}}, 1\right) .
$$

If we choose our units so that $\tilde{F}(1,1)=1$, then we have the nice property that $k=k_{i}$ implies that $y=y_{i}$. Therefore, we can think of technique $i$ as being indexed by $a_{i}$ and $b_{i}$, or, equivalently, by $k_{i}$ and $y_{i}$.

The shape of the global production function is driven by the distribution of alternative production techniques rather than by the shape of the local production function that applies for a single technique. ${ }^{2}$ To illustrate this, consider the example given in Figure 1. The circles in this figure denote different production techniques that are available - the set of $\left(k_{i}, y_{i}\right)$ pairs. For a subset of these, we also plot the local production function $y=\tilde{F}\left(b_{i} k, a_{i}\right)$. Finally, the heavy solid line shows the global production function, given by the convex hull of the local production techniques. For any given level of $k$, the global production function shows the maximum amount of output per worker that can be produced using the set of ideas that are available.

\footnotetext{
${ }^{2}$ Other models in the literature feature a difference between the short-run and long-run elasticities of substitution, as opposed to the local-global distinction made here. These include the putty-clay models of Caballero and Hammour (1998) and Gilchrist and Williams (2000).
} 
FIGURE 1. An Example of the Global Production Function

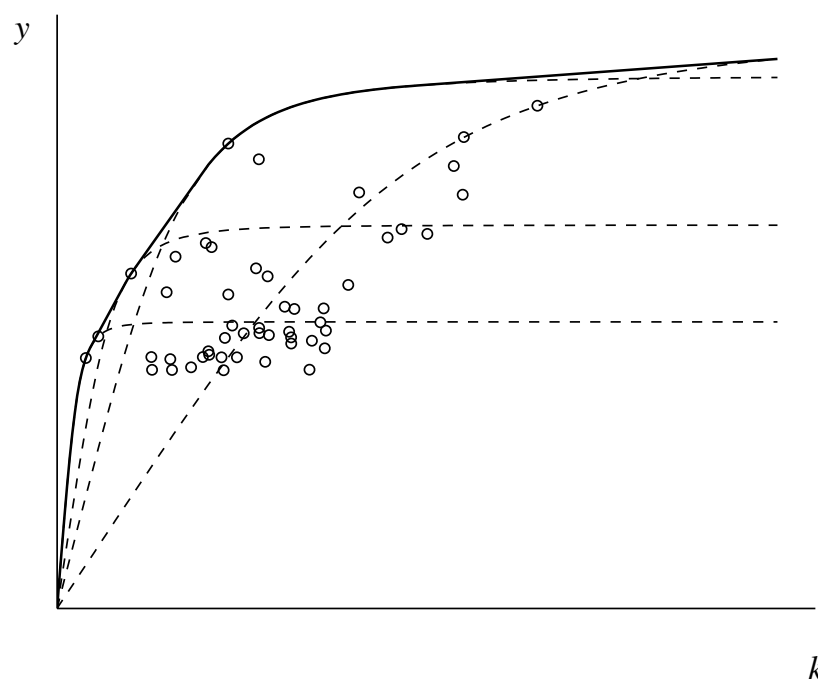

Circles identify distinct production techniques; for some of these, the local production function associated with the technique has been drawn as a dashed line. The heavy solid line shows the convex hull of the local production functions, i.e. the global production function.

The key question we'd like to answer is this: What is the shape of the global production function? To make progress, we now turn to a simple baseline model.

\subsection{The Baseline Model}

We begin with a simple model, really not much more than an example. However this baseline model turns out to be very useful: it is easy to analyze and captures the essence of the model with more detailed microfoundations that is presented in Section 3.

At any given point in time, a firm (or the economy) has a stock of ideas from which to choose. To characterize the global production function, 
notice that for each point in the input space, the economy will choose to produce with the idea that yields the largest amount of output.

To make this problem more precise, suppose $N$ is the cumulative amount of research the firm has undertaken, and suppose this research has generated a menu of technology choices given by

$$
b=H(a, N)
$$

where $H_{a}<0$ and $H_{N}>0$. From its research effort, $N$, the firm discovers a set of ideas. The frontier of this set is a technology menu that involves a tradeoff between ideas with a high level of $a$ and ideas with a high level of $b$. As more research is conducted, $N$ rises, the firm discovers more ideas, and this menu shifts out.

The firm's problem then is to choose $a$ and $b$ from the technology menu to maximize the level of production for any given set of inputs, and this defines the global production function:

$$
Y=F(K, L ; N) \equiv \max _{b, a} \tilde{F}(b K, a L)
$$

subject to (5), assuming $K, L$, and $N$ are given. As discussed above, we assume that $\tilde{F}(\cdot, \cdot)$ has an elasticity of substitution less than one and exhibits constant returns to scale in $K$ and $L$.

Notice that the problem here is to choose the levels of $a$ and $b$. Related problems appear in the literature on the direction of technical change; see Acemoglu (2003a), Kennedy (1964), Samuelson (1965) and Drandakis and Phelps (1966). However, in these problems the choice variables and the constraints are typically expressed in terms of the growth rates of $a$ and $b$ rather than the levels, resulting in a conceptually different problem. There, the notion is that one can choose whether to discover ideas with a high $a$ or a high $b$. Here, the notion is that one discovers ideas in undirected search, and that each idea is an $(a, b)$ pair. 
FIGURE 2. Direction of Technical Change

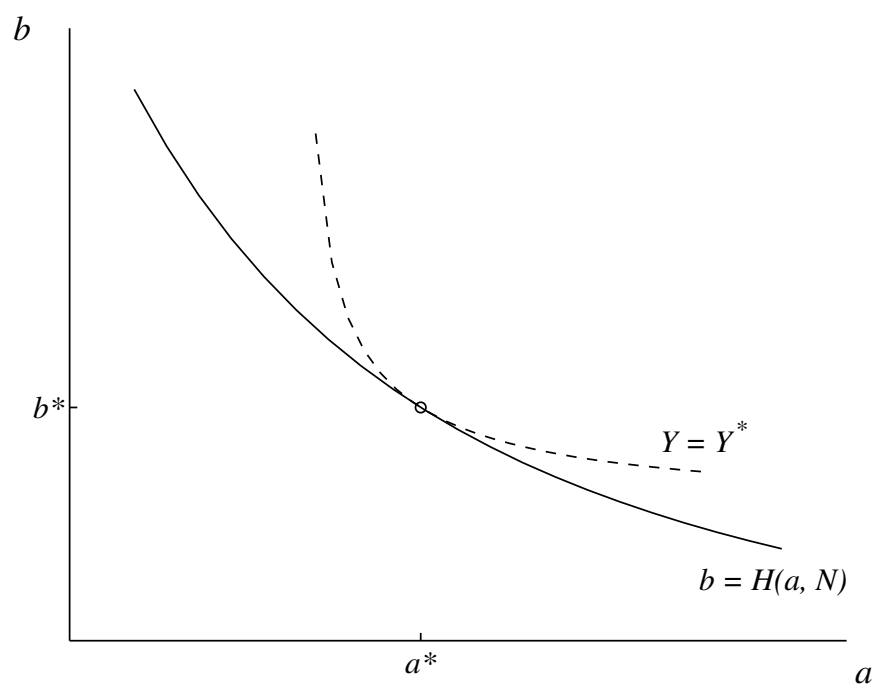

The solution to this problem is straightforward. Graphically, it is shown in Figure 2. Algebraically, an interior solution equates the ratio of the labor and capital shares to the elasticity of the $H$ curve:

$$
\frac{1-\theta_{K}}{\theta_{K}}=-\frac{\partial H}{\partial a} \frac{a}{H} \equiv \eta_{H a}
$$

where $\theta_{K}(a, b ; K, L) \equiv \tilde{F}_{1} b K / Y$.

In Figure 2, we drew the technology menu as convex to the origin. Of course, this is just for illustrative purposes; we could have drawn the curve as concave or linear. However, it turns out that the constant elasticity version of the convex curve delivers a particularly nice result. ${ }^{3}$ In particular, suppose the constraint is given by

$$
b=N a^{-\eta}, \quad \eta>0 .
$$

\footnotetext{
${ }^{3}$ In this case, the assumption that $\tilde{F}$ has an elasticity of substitution less than one guarantees that the iso-output curves are more sharply curved that the technology menu, producing an interior solution.
} 
In this case, the elasticity $\eta_{H a}=\eta$ is constant, so the choice of the technology levels leads to a first-order condition that sets the capital share equal to the constant $1 / 1+\eta$.

The constancy of the capital share then leads to two useful and interesting results. First, the global production function takes a Cobb-Douglas form: for any levels of the inputs $K$ and $L$, and any location of the technology frontier, $N$, the choice of technology leads the elasticity of output with respect to capital and labor to be constant.

In fact, it is easy to derive the exact form of the global production function by combining the local-global insights of Section 2.1 with the technology menu. For some technique $i$, recall the equivalent ways we have of describing the technique:

$$
\begin{aligned}
y_{i} & \equiv a_{i} \\
k_{i} & \equiv \frac{a_{i}}{b_{i}}
\end{aligned}
$$

From the technology frontier in equation (8), we know that $b_{i}$ and $a_{i}$ are related by $b_{i}=N a_{i}^{-\eta}$. Simple algebra shows that $y_{i}$ and $k_{i}$ are therefore related by

$$
y_{i}=\left(N k_{i}\right)^{1 / 1+\eta}
$$

That is, given the constant elasticity form of the technology frontier, a plot of the techniques in $(k, y)$ space like that in Figure 1 yields a Cobb-Douglas production function. With this continuous formulation for the frontier, the global production function is exactly equal to the technology frontier in $(k, y)$ space. ${ }^{4}$ Multiplying by $L$ to get back to the standard form, the global

\footnotetext{
${ }^{4}$ For this to be true, we need the local production techniques to paste up smoothly with the global production function. For example, if $\tilde{F}$ is a CES function with a capital share parameter $\lambda$ (see, for example, equation (33) below), the global production function is actually proportional to that in equation (12). To make the factor of proportionality equal to one, we need $\lambda=1 / 1+\eta$, so that the factor share at $k=k_{i}$ is exactly $1 / 1+\eta$.
} 
production function is given by

$$
Y=N^{\theta} K^{\theta} L^{1-\theta}
$$

where $\theta \equiv 1 / 1+\eta$.

The second key result is related to the direction of technical change. To see this, consider embedding this production setup in a standard neoclassical growth model. ${ }^{5}$ The fact that the global production function is CobbDouglas implies immediately that such a model will exhibit a balanced growth path with positive growth provided $N$ grows exponentially.

The fact that such a model possesses a balanced growth path turns out to have a strong implication for the direction of technical change. This is just an application of the Steady-State Growth Theorem: If a neoclassical growth model exhibits steady-state growth with a nonzero capital share, then either the production function is Cobb-Douglas or technical change is labor augmenting. ${ }^{6}$ Since the local production function is not Cobb-Douglas (and production always occurs with some local production function), this must mean that in the long run, technical change is purely labor augmenting and $b$ is constant. Moreover, the fact that the capital share equals $1 / 1+\eta$ implies that the level of $b$ is chosen so that the capital share is invariant to the capital-output ratio, one of the key results in Acemoglu (2003b).

What is the intuition for the result that technical change is purely labor augmenting? Notice that in steady state $K / Y$ must be constant. To have a positive capital share, then, the marginal product of capital must also be

\footnotetext{
${ }^{5}$ By this we mean the usual Ramsey-Cass-Koopmans model with isoelastic utility, constant population growth, and constant growth in $N$.

${ }^{6}$ For a discussion and proof of this theorem, see Barro and Sala-i-Martin (1995), Chapter 2. The nonzero capital share qualification is important. For example, a neoclassical growth model with a CES production function $Y=F(B K, A L)$ with an elasticity of substitution less than one and with exponential growth in $A$ and $B$ does have a balanced growth path. Asymptotically, production behaves as if there were an infinite amount of effective capital so that $Y=A L$.
} 
positive and constant. An increase in $b$ has two effects: first, it raises the marginal product of capital directly. But second, it increases the amount of effective capital, driving down the marginal product. In the Cobb-Douglas case, these two effects cancel, but otherwise one or the other dominates, imparting a trend to the marginal product unless $b$ is constant. ${ }^{7}$

The insight from this simple example is that if the technology frontier i.e. the way in which the levels of $a$ and $b$ trade off - exhibits a constant elasticity, then the global production function will be Cobb-Douglas and technological change will be labor-augmenting in the long run. But is there any reason to think that the technology frontier takes this particular shape?

\section{MICROFOUNDATIONS: PARETO DISTRIBUTIONS}

The baseline model is straightforward and yields strong predictions. However, it involves a very particular specification of the technology menu. It turns out that this specification can be derived from a model of ideas with substantially richer microfoundations. This is the subject of the current section. ${ }^{8}$

\subsection{Setup}

Let $i=0,1, \ldots$ index the production techniques - the ideas - that are available at a given point in time. The production technique associated

\footnotetext{
${ }^{7}$ Another intuition is the following. Notice that $Y=\tilde{F}(b K, a L)$ has an elasticity of substitution between effective capital and effective labor that is less than one. Therefore, $K$ and $a$ are relative complements while $b$ and $K$ are relative substitutes (the elasticity of substitution between $b$ and $K$ is equal to one). Capital accumulation substitutes for growth in $b$ while it complements and reinforces growth in $a$.

${ }^{8}$ I owe a large debt to Sam Kortum in this section. A previous version of this paper contained a much more cumbersome derivation of the Cobb-Douglas result that applied asymptotically as research effort gets large. Kortum, in discussing this earlier version at a conference, developed the more elegant and useful Poisson approach that follows. The asymptotic approach gets by without making the Poisson assumption, while the Poisson approach is more tractable and delivers a result that applies for finite research effort. The asymptotic result that drops the Poisson assumption is developed in the Appendix.
} 
with idea $i$ is $\tilde{F}\left(b_{i} K, a_{i} L\right)$. Because it results in a more tractable problem that yields analytic results, we make the extreme assumption that this local production technology is Leontief:

$$
Y=\tilde{F}\left(b_{i} K, a_{i} L\right)=\min \left\{b_{i} K, a_{i} L\right\}
$$

Of course, the intuition regarding the global production function suggests that it is determined by the distribution of ideas, not by the shape of the local production function. In later simulation results, we confirm that the Leontief assumption can be relaxed.

New ideas for production are discovered through research. A single research endeavor yields a number of ideas drawn from a Poisson distribution with a parameter normalized to one. In expectation, then, each research endeavor yields one idea. Let $N$ denote the cumulative number of research endeavors that have been undertaken. Then the number of ideas, $n$ that have been discovered as a result of these $N$ attempts is a random variable drawn from a Poisson distribution with parameter $N$.

The discovery of an idea results in a new production technique, described by its labor-augmenting and capital-augmenting parameters $a_{i}$ and $b_{i}$. These parameters are drawn from a joint distribution given by

$$
\operatorname{Prob}\left[b_{i}>b, a_{i}>a\right]=G(b, a),
$$

where the support for this distribution is $a_{i} \geq \gamma_{a}>0$ and $b_{i} \geq \gamma_{b}>0$. We specify this distribution in its complementary form because this simplifies some of the equations that follow.

\subsection{Deriving the Production Function}

The global production function describes, as a function of inputs, the maximum amount of output that can be produced using any combination of existing production techniques. We have already made one simplification 
in our setup by limiting consideration to Leontief techniques. Now we make another by ignoring combinations of techniques and allowing only a single technique to be used at each point in time. Again, this is a simplifying assumption that allows for an analytic result, but it will be relaxed later in the numerical simulations.

Definition 3.1. The global production function $F(K, L ; n)$ is given as

$$
F(K, L ; n) \equiv \max _{i \in\{0, \ldots, n-1\}} \tilde{F}\left(b_{i} K, a_{i} L\right)
$$

We build up to characterizing this object through several steps. First, consider particular levels of the inputs, $K$ and $L$. Let $Y_{i}(K, L) \equiv \tilde{F}\left(b_{i} K, a_{i} L\right)$ denote output using technique $i$. Then, since $\tilde{F}$ is Leontief, the distribution of $Y_{i}$ is given by ${ }^{9}$

$$
\begin{aligned}
\operatorname{Prob}\left[Y_{i}>\tilde{y}\right] & =\operatorname{Prob}\left[b_{i} K>\tilde{y}, a_{i} L>\tilde{y}\right] \\
& =G(\tilde{y} / K, \tilde{y} / L) .
\end{aligned}
$$

Next, suppose there are $n$ ideas that have been discovered. In this case, the output level associated with the global production function is distributed as

$$
\operatorname{Prob}\left[\max _{i}\left\{Y_{i}\right\} \leq \tilde{y}\right]=(1-G(\tilde{y} / K, \tilde{y} / L))^{n}
$$

At this point, we can use the nice properties of the Poisson distribution to make further progress. Recall that $n \sim \operatorname{Poisson}(N)$, so as a function of the total number of research attempts, $N$, we have

\footnotetext{
${ }^{9}$ Since $b_{i} \geq \gamma_{b}$ and $a_{i} \geq \gamma_{a}$, the support for this distribution is $\tilde{y} \geq \max \left\{\gamma_{b} K, \gamma_{a} L\right\}$.
} 


$$
\begin{aligned}
\operatorname{Prob}[\max \{ & \left.\left.Y_{i}\right\} \leq \tilde{y}\right] \\
& =\sum_{n=0}^{\infty} \frac{e^{-N} N^{n}}{n !}(1-G(\tilde{y} / K, \tilde{y} / L))^{n} \\
& =e^{-N} \sum_{n=0}^{\infty} \frac{(N(1-G(\tilde{y} / K, \tilde{y} / L)))^{n}}{n !} \\
& =e^{-N} \cdot e^{N(1-G(\cdot))} \\
& =e^{-N G(\tilde{y} / K, \tilde{y} / L)} .
\end{aligned}
$$

For a general joint distribution function $G$, this last equation describes the distribution of the global production function when cumulative research effort is $N .^{10}$

To go further, we now make a key assumption about the distribution of ideas:

Assumption 3.1. Ideas are drawn from independent Pareto distributions:

$$
\begin{aligned}
& \operatorname{Prob}\left[a_{i} \leq a\right]=1-\left(\frac{a}{\gamma_{a}}\right)^{-\alpha}, a \geq \gamma_{a}>0 \\
& \operatorname{Prob}\left[b_{i} \leq b\right]=1-\left(\frac{b}{\gamma_{b}}\right)^{-\beta}, \quad b \geq \gamma_{b}>0,
\end{aligned}
$$

where $\alpha>0, \beta>0$, and $\alpha+\beta>1 .^{11}$

\footnotetext{
${ }^{10}$ See Proposition 2.1 in Kortum (1997) for this style of reasoning, i.e. for an approach that uses a Poisson process to get an exact extreme value distribution that is easy to work with rather than an asymptotic result. Also Johnson, Kotz, and Balakrishnan, Continuous Univariate Distributions, Volume 2, pp. 11 and 91-92.

${ }^{11}$ This last condition that the sum of the two parameters be greater than one is needed so that the mean of the Fréchet distribution below exists. On a related point, recall that for a Pareto distribution, the $k$ th moment exists only if the shape parameter (e.g. $\alpha$ or $\beta$ ) is larger than $k$.
} 
With this assumption, the joint distribution of $a_{i}$ and $b_{i}$ satisfies

$$
\operatorname{Prob}\left[b_{i}>b, a_{i}>a\right]=G(b, a)=\left(\frac{b}{\gamma_{b}}\right)^{-\beta}\left(\frac{a}{\gamma_{a}}\right)^{-\alpha} .
$$

And therefore,

$$
\operatorname{Prob}\left[Y_{i}>\tilde{y}\right]=G(\tilde{y} / K, \tilde{y} / L)=\gamma K^{\beta} L^{\alpha} \tilde{y}^{-(\alpha+\beta)}
$$

where $\gamma \equiv \gamma_{a}^{\alpha} \gamma_{b}^{\beta}$. That is, the distribution of $Y_{i}$ is itself Pareto.

The final step is to combine the Pareto assumption with the global production function result derived in equation (18). It is straightforward to show that the distribution of the output that can be produced with the global production function, given inputs of $K$ and $L$, is

$$
\operatorname{Prob}\left[\max \left\{Y_{i}\right\} \leq \tilde{y}\right]=e^{-\gamma N K^{\beta} L^{\alpha} \tilde{y}^{-(\alpha+\beta)}} .
$$

This distribution is known as a Fréchet distribution. ${ }^{12}$

Finally, taking expectations over this distribution, one sees that expected output, given $N$ cumulative research draws and inputs $K$ and $L$, is given by

$$
E[Y] \equiv E\left[\max Y_{i}\right]=\mu\left(\gamma N K^{\beta} L^{\alpha}\right)^{\frac{1}{\alpha+\beta}}
$$

where $\mu \equiv \Gamma(1-1 /(\alpha+\beta))$ is a constant that depends on Euler's factorial function. ${ }^{13}$

One can also use the distribution in equation (23) to write the level of output as a random variable: ${ }^{14}$

$$
Y=\left(\gamma N K^{\beta} L^{\alpha}\right)^{\frac{1}{\alpha+\beta}} \epsilon
$$

\footnotetext{
${ }^{12}$ See Kortum (1997), Galambos (1978), and Castillo (1988) for more.

${ }^{13}$ Surprisingly few of the reference books on extreme value theory actually report the mean of the Fréchet distribution. For a distribution function $F(x)=\exp \left(-((x-\lambda) / \delta)^{-\beta}\right)$, Castillo (1988) reports that the mean is $\lambda+\delta \Gamma(1-1 / \beta)$ for $\beta>1$.

${ }^{14}$ In particular, notice that $\operatorname{Prob}[Y / z \leq \tilde{y}]=\exp \left(-\tilde{y}^{-(\alpha+\beta)}\right)$ where $z \equiv\left(\gamma K^{\beta} L^{\alpha}\right)^{1 / \alpha+\beta}$.
} 
where $\epsilon$ is a random variable drawn from a Fréchet distribution with shape parameter $\alpha+\beta$ and a scale parameter equal to unity.

The Poisson structure for the arrival of ideas is a convenient device for simplifying the presentation of the Cobb-Douglas result, but it is not necessary. The Appendix derives the Cobb-Douglas production function as an asymptotic result when the number of ideas gets large.

\section{DISCUSSION}

The result given in equation (25) is one of the main results in the paper. If ideas are drawn from Pareto distributions, then the global production function takes, at least in expectation, the Cobb-Douglas form. For any given production technique, a firm may find it difficult to substitute capital for labor and vice versa, leading the curvature of the production function to set in quickly. However, when firms are allowed to switch between production technologies, the global production function depends on the distribution of ideas. If that distribution happens to be a Pareto distribution, then the production function is Cobb-Douglas.

We can now make a number of remarks. First, the exponent in the CobbDouglas function depends directly on the parameters of the Pareto search distributions. The easier it is to find ideas that augment a particular factor, the lower is the relevant Pareto parameter (e.g. $\alpha$ or $\beta$ ), and the lower is the exponent on that factor. Intuitively, better ideas on average reduce factor shares because the elasticity of substitution is less than one. Some additional remarks follow.

\subsection{Relationship to the Baseline Model}

The simple baseline model given at the beginning of this paper postulated a technology menu and showed that if this menu exhibited a constant elasticity, then one could derive a Cobb-Douglas global production function. 
The model with microfoundations based on Pareto distributions turns out to deliver a stochastic version of this technology menu.

In the model, the stochastic version of this menu can be seen by considering an iso-probability curve $\operatorname{Prob}\left[b_{i}>b, a_{i}>a\right] \equiv G(b, a)=C$, where $C>0$ is some constant. With the joint Pareto distribution, this iso-probability curve is given by

$$
b=\left(\frac{\gamma}{C}\right)^{1 / \beta} a^{-\alpha / \beta}
$$

This iso-curve has a constant elasticity equal to $-\alpha / \beta$ and shifts up as the probability $C$ is lowered.

In terms of the baseline model, the Pareto distribution therefore delivers $\eta_{H a}=\alpha / \beta$. The first-order condition in equation (7) then implies that technologies will be chosen so that the capital share is equal to $\beta /(\alpha+$ $\beta$ ), which is exactly the exponent in the global Cobb-Douglas production function of the Pareto model, as shown in equation (25).

\subsection{Houthakker (1955-1956)}

The notion that Pareto distributions, appropriately "kicked," can deliver a Cobb-Douglas production function is a classic result by Houthakker (19551956). Houthakker considers a world of production units (e.g. firms) that produce with Leontief technologies where the Leontief coefficients are distributed across firms according to a Pareto distribution. Importantly, each firm has limited capacity, so that the only way to expand output is to use additional firms. Houthakker then shows that the aggregate production function across these units is Cobb-Douglas.

The result here obviously builds directly on Houthakker's insight that Pareto distributions can generate Cobb-Douglas production functions. The result differs from Houthakker's in several ways, however. First, Houthakker's result is an aggregation result. Here, in contrast, the result applies at the 
level of a single production unit (be it a firm, industry, or country). Second, the Leontief restriction in Houthakker's paper is important for the result; it allows the aggregation to be a function only of the Pareto distributions. Here, in contrast, the result is really about the shape of the global production function, looking across techniques. The local shape of the production function does not really matter. This was apparent in the simple baseline model given earlier, and it will be confirmed numerically in Section 6 .

Finally, Houthakker's result relies on the presence of capacity constraints. If one wants to expand output, one has to add additional production units, essentially of lower "quality." Because of these capacity constraints, his aggregate production function is characterized by decreasing returns to scale. In the context of an idea model, such constraints are undesirable: one would like to allow the firm to take its best idea and use it for every unit of production. That is, one would like the setup to respect the nonrivalry of ideas and the replication argument for constant returns, as is true in the formulation here. ${ }^{15}$

\subsection{Evidence for Pareto Distributions?}

The next main comment is that Pareto distributions are crucial to the result. Is it plausible that the distributions for ideas are Pareto?

In terms of direct evidence, there are a number of references related to patents, profitability, and citations. First, it is worth noting that many of the tests in this literature are about whether or not the relevant variable obeys a Pareto distribution. That is, Pareto serves as a benchmark. In terms of findings, this literature either supports the Pareto distribution or

\footnotetext{
${ }^{15}$ Lagos (2004) embeds the Houthakker formulation in a Mortenson-Pissarides search model to provide a theory of total factor productivity differences. In his setup, firms (capital) match with labor and have a match quality that is drawn from a Pareto distribution. Capital is the quasi-fixed factor so that the setup generates constant returns to scale in capital and labor. Nevertheless, because each unit of capital gets its own Pareto draw, a firm cannot expand production by increasing its size at its best match quality.
} 
finds that it is difficult to distinguish between the Pareto and the lognormal distributions. For example, Harhoff, Scherer and Vopel (1997) examine the distribution of the value of patents in Germany and the United States. For patents worth more than $\$ 500,000$ or more than 100,000 Deutsche Marks, a Pareto distribution accurately describes patent values, although for the entire range of patent values a lognormal seems to fit better. Bertran (2003) finds evidence of a Pareto distribution for ideas by using patent citation data to value patents. Grabowski (2002) produces a graph of the present discounted value of profits for new chemical entities by decile in the pharmaceutical industry for 1990-1994 that supports a highly-skewed distribution. Sornette and Zajdenweber (2000) interpret earlier similar work by Grabowski and Vernon as evidence in favor of a Pareto distribution.

Lotka (1926), a classic reference on scientific productivity, shows that the distribution of scientific publications per author is Pareto. This result appears to have stood the test of time across a range of disciplines, even in economics, as shown by Cox and Chung (1991). Huber (1998) looks for this result among inventors and finds some evidence that the distribution of patents per inventor is also Pareto, although the sample is small. Somewhat further removed but still related, evidence of Pareto distributions is found by Sornette and Zajdenweber (1999) for world movie revenues and by Chevalier and Goolsbee (2004) for book sales.

In addition to the direct evidence, there are also conceptual reasons to think ideas might come from Pareto distributions. To begin, consider a simple example. Imagine drawing social security numbers for the U.S. population at random, and for each person drawn, record their income and their height. Also keep track of the largest draw to date: let $y_{z}^{\max }$ denote the maximum income and let $h_{z}^{\max }$ denote the maximum height after $z$ draws have been made. Now consider the following conditional probability: $\operatorname{Prob}\left\{X \geq \gamma x^{\max } \mid X \geq x^{\max }\right\}$ for $\gamma>1$, where $x$ stands 
for either income or height. This probability answers the question: "Given that the tallest person observed so far is 6 feet 6 inches tall and given that we just found someone even taller, what is the probability that this new person is more than 5 percent taller than our 6 foot 6 inch person?" Clearly as $h^{\text {max }}$ gets larger and larger, this conditional probability gets smaller and smaller - there is no one in the world taller than ten feet.

In contrast, consider the income draws. Now, the probability answers the question: "Given that the highest-earning person observed so far has an annual income of $\$ 240,000$ and given that we just found someone who earns even more, what is the probability that this new person's earnings exceed the previous maximum by more than 5 percent?" It turns out empirically that in the case of incomes, this probability does not depend on the level of $y^{\max }$ being considered. Indeed, it was exactly this observation on incomes that led Pareto to formulate the distribution that bears his name: the defining characteristic of the Pareto distribution is that the conditional probability given above is invariant to $x^{\max } \cdot{ }^{16}$

In applying this example to growth models, one is led to ask whether the distribution of ideas is more like the distribution of heights or the distribution of incomes. An important insight into this question was developed by Kortum (1997). Kortum formulates a growth model where productivity levels (ideas) are draws from a distribution. He shows that this model generates steady-state growth only if the distribution has Pareto tails. That is, what the model requires is that the probability of finding an idea that is 5 percent better than the current best idea is invariant to the level of productivity embodied in the current best idea. Of course, this is almost the very definition of a steady state: the probability of improving economywide productivity by 5 percent can't depend on the level of productivity.

\footnotetext{
${ }^{16}$ Saez (2001) shows this invariance for the United States in 1992 and 1993 for incomes between $\$ 100,000$ and $\$ 30$ million.
} 
This requirement is satisfied only if the upper tail of the distribution is a power function, i.e. only if the upper tail is Pareto. ${ }^{17}$

A literature in physics on "scale invariance" suggests that if a stochastic process is to be invariant to scale, it must involve Pareto distributions. Steady-state growth is simply a growth rate that is invariant to scale (defined in this context as the initial level of productivity). Whether incomes are at 100 or 1000 , steady-state growth requires the growth rate to be the same in both cases.

Additional insight into this issue emerges from Gabaix (1999). Whereas Kortum shows that Pareto distributions lead to steady-state growth, Gabaix essentially shows the reverse in his explanation of Zipf's Law for the size of cities. He assumes that city sizes grow at a common exponential rate plus an idiosynchratic shock. He then shows that this exponential growth generates a Pareto distribution for city sizes. ${ }^{18}$

These papers by Kortum and Gabaix suggest that Pareto distributions and exponential growth are really just two sides of the same coin. The result in the present paper draws out this connection further and highlights the additional implication for the shape of production functions. Not only are Pareto distributions necessary for exponential growth, but they also imply that the global production function takes a Cobb-Douglas form.

\footnotetext{
${ }^{17}$ Kortum also shows that if the tails of the distribution are thinner than Pareto, as is the case for the log normal or exponential distributions, then exponential growth rates decline to zero. If the tails are thicker, then presumably growth rates rise over time, but this case is not analyzed by Kortum.

${ }^{18} \mathrm{An}$ important additional requirement in the Gabaix paper is that there be some positive lower bound to city sizes that functions as a reflecting barrier. Otherwise, for example, normally distributed random shocks results in a log-normal distribution of city cizes. Alternatively, if the length of time that has passed since each city was created is a random variable with an exponential distribution, then no lower bound is needed and one recovers the Pareto result. See Mitzenmacher (2003) for a direct discussion of these alternatives, as well as Cordoba (2003) and Rossi-Hansberg and Wright (2004).
} 


\section{THE DIRECTION OF TECHNICAL CHANGE}

The second main result of the paper is related to the direction of technical change. It turns out that this same setup, when embedded in a standard neoclassical growth model, delivers the result that technological change is purely labor augmenting in the long run. That is, even though the largest value of $b_{i}$ associated with any idea goes to infinity, this Pareto-based growth model delivers the result that $a_{i}(t)$ grows on average while $b_{i}(t)$ is stationary.

To see this result, we first embed our existing setup in a standard neoclassical growth model. The production side of the model is exactly as specified in Section 3. Capital accumulates in the usual way, and we assume the investment rate $s$ is a constant:

$$
K_{t+1}=(1-\delta) K_{t}+s Y_{t}, \delta, s \in(0,1)
$$

Finally, we assume that the cumulative number of research endeavors as of date $t, N_{t}$, grows exogenously at rate $g>0$ :

$$
N_{t}=N_{0} e^{g t}
$$

As in Jones (1995) and Kortum (1997), one natural interpretation of this assumption is that research endeavors are undertaken by researchers, and $g$ is proportional to population growth. ${ }^{19}$

For this model, we have already shown that the global production function is given by

$$
Y_{t}=\left(\gamma N_{t} K_{t}^{\beta} L_{t}^{\alpha}\right)^{\frac{1}{\alpha+\beta}} \epsilon_{t}
$$

\footnotetext{
${ }^{19}$ For example, one could have $\Delta N_{t+1}=R_{t}^{\lambda} N_{t}^{\phi}$, where $R_{t}$ represents the number of researchers working in period $t$. In this case, if the number of researchers grows at a constant exponential rate, then the growth rate of $N$ converges to a constant that is proportional to this population growth rate. Of course, one logical case to consider is where $\lambda=1$ and $\phi=0$, so that the number of research endeavors in a period is just proportional to the number of researchers.
} 
It is then straightforward to show that the average growth rate of output per worker $y$ in the model in a stationary steady state is given by ${ }^{20}$

$$
E\left[\log \frac{y_{t+1}}{y_{t}}\right] \approx g / \alpha .
$$

The growth rate of output per worker is proportional to the rate of growth of research effort. The factor of proportionality depends only on the search parameter of the Pareto distribution for the labor-augmenting ideas. In particular, the easier it is to find higher $a_{i}$, the faster is the average rate of economic growth.

The fact that this growth rate depends on $\alpha$ but not on $\beta$ is the first clue that there is something further to explore here: if it is easier to find better labor-augmenting ideas, the average growth rate is higher, but if it is easier to find better capital-augmenting ideas, the average growth rate is unaffected.

To understand this fact, it is helpful to look back at the local production function. Even though the global production function is Cobb-Douglas, production at some date $t$ always occurs with some technique $i(t)$ :

$$
Y_{t}=\tilde{F}\left(b_{i(t)} K_{t}, a_{i(t)} L_{t}\right) \text {. }
$$

Now recall the Steady-State Growth Theorem discussed earlier: If a neoclassical growth model exhibits steady-state growth with a nonzero capital share, then either the production function is Cobb-Douglas or technical change is labor augmenting. In this case, the (local) production function is not Cobb-Douglas and we do have a (stationary) steady state. The implication is that technical change must be labor-augmenting in the model.

\footnotetext{
${ }^{20}$ Rewriting the production function in per worker terms, one has

$$
\log \frac{y_{t+1}}{y_{t}}=\frac{1}{\alpha+\beta} \log \frac{N_{t+1}}{N_{t}}+\frac{\beta}{\alpha+\beta} \log \frac{k_{t+1}}{k_{t}}+\log \frac{\epsilon_{t+1}}{\epsilon_{t}} .
$$
}

Taking expectations of this equation and equating the growth rates of $y$ and $k$ yields the desired result. 
That is, despite the fact that $\max _{i} b_{i} \rightarrow \infty$ as $t \rightarrow \infty$, the time path for $b_{i(t)}$ - i.e. the time path of the $b_{i}$ 's associated with the ideas that are actually used - must have an average growth rate equal to zero in the limit. The intuition is the same as in the simple baseline model: $K$ and $b$ are relative substitutes, while $K$ and $a$ are relative complements in production. This means that capital accumulation leads the economy to increase $a$ at the expense of a stable $b .^{21}$

\section{SIMULATION RESULTS}

We now turn to a full simulation based on the Pareto model. In addition to providing an illustration of the results, we take this opportunity to relax the Leontief assumption on the local production function. Instead, we assume the local production function takes the CES form:

$$
Y_{t}=\tilde{F}\left(b_{i} K_{t}, a_{i} L_{t}\right)=\left(\lambda\left(b_{i} K_{t}\right)^{\rho}+(1-\lambda)\left(a_{i} L_{t}\right)^{\rho}\right)^{1 / \rho},
$$

where $\rho<0$ so that the elasticity of substitution is $\sigma \equiv \frac{1}{1-\rho}<1$. We also allow production units to use two production techniques at a time in order to convexify the production set, analogous to the picture given at the beginning of the paper in Figure 1.

The remainder of the model is as specified before. Apart from the change to the CES function, the production setup is the same as that given in Section 3 and the rest of the model follows the constant saving setup of Section 5 .

We begin by showing that the CES setup still delivers a Cobb-Douglas global production function, at least on average. For this result, we repeat

\footnotetext{
${ }^{21}$ This result leads to an important observation related to extending the model. Recall that with the Pareto assumption, $\gamma_{b}$ is the smallest value of $b$ that can be drawn, and similarly $\gamma_{a}$ is the smallest value of $a$ that can be drawn. Now consider allowing these distributions to shift. There seems to be no obstacle to allowing for exponential shifts in $\gamma_{a}$ over time. However, increases in $\gamma_{b}$ turn out to lower the capital share in the model. If $\gamma_{b}$ were to rise exponentially, the capital share would be driven toward zero, on average.
} 
FIGURE 3. The Cobb-Douglas Result

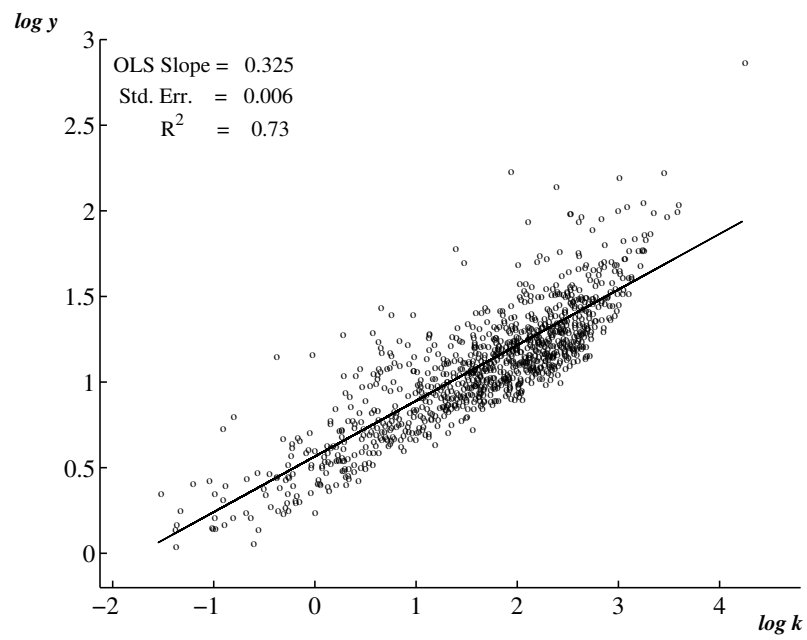

Note: The figure shows 1000 capital-output combinations from the global production function. The parameter values used in the simulation are $N=500$, $\alpha=5, \beta=2.5, \gamma_{a}=1, \gamma_{b}=0.2$, and $\rho=-1$.

the following set of steps to obtain 1000 capital-output pairs: We first set cumulative research effort $N$ to 500 , so that on average there are 500 ideas in each iteration. We compute the convex hull of the CES functions associated with these ideas to get a global production function. ${ }^{22}$ Next, we choose a level of capital per worker $k$ randomly from a uniform distribution between the smallest value of $k_{i}$ and the largest value of $k_{i}$ for the iteration. Finally, we record the output of the global production function associated with this input.

Following this procedure yields a graph like that shown in Figure 3. The key parameter values in this simulation are $\alpha=5$ and $\beta=2.5$, so that

\footnotetext{
${ }^{22}$ Computing the convex hull of the overlapping CES production functions is a computationally intensive problem, especially when the number of ideas gets large. To simplify, we first compute the convex hull of the $\left(k_{i}, y_{i}\right)$ points. Then, we compute the convex hull of the CES functions associated with this limited set of points. To approximate the CES curve, we divide the capital interval into 100 equally-spaced points.
} 
the theory suggests we should expect a Cobb-Douglas production function with a capital exponent of $\beta / \alpha+\beta=1 / 3{ }^{23}$ As the figure shows, the relation between $\log y$ and $\log k$ is linear, with a slope that is very close to this value.

We next consider a simulation run for the full dynamic time path of the Pareto model. Continuing with the parameter choices already made, we additionally assume $g=.10$, which implies an annual growth rate of 2 percent for output per worker in the steady state. We simulate this model for 100 years and plot the results in several figures. ${ }^{24}$ Figure 4 shows a subset of the more than 1 million techniques that are discovered over these 100 periods. In particular, we plot only the 300 points with the highest values of $y$ (these are shown with circles "o"). Without this truncation, the lower triangle in the figure that is currently blank but for the plus signs is filled in as solid black. In addition, the capital-output combinations that are actually used in each period are plotted with a plus sign (“+”). When a single technique is used for a large number of periods, the points trace out the CES production function. Alternatively, if the economy is convexifying by using two techniques, the points trace out a line. Finally, when the economy switches to a new technique, the capital-output combinations jump upward.

Figure 5 shows output per worker over time, plotted on a log scale. The

\footnotetext{
${ }^{23}$ The standard capital share pins down the ratio of $\alpha / \beta$, but it does not tell us the basic scale of these parameters. The studies cited earlier related to patent values and scientific productivity typically find Pareto parameters that are in the range of 0.5 to 1.5 . We have chosen higher values here for illustration. The following exercise is helpful in thinking about this: What is the median value of a productivity draw, conditional on that draw being larger than some value, $x$ ? If $\alpha$ is the Pareto parameter, then the answer to this question turns out to be $2^{1 / \alpha} x \approx(1+0.7 / \alpha) x$. For example, if $\alpha=2$, then the median value, conditional on a draw being higher than $x$, is about $1.4 x$. This says that the average idea that exceeds the frontier exceeds it by 40 percent. This implies very large jumps, which might be plausible at the micro level but seem too large at the macro level. A value of $\alpha=5$ instead gives an average jump of about 14 percent, which is still somewhat large. Aggregation would, one suspects, smooth these jumps out.

${ }^{24}$ Additional parameter values used in the simulation are listed in the notes to Figure 4.
} 
FIGURE 4. Production in the Simulated Economy

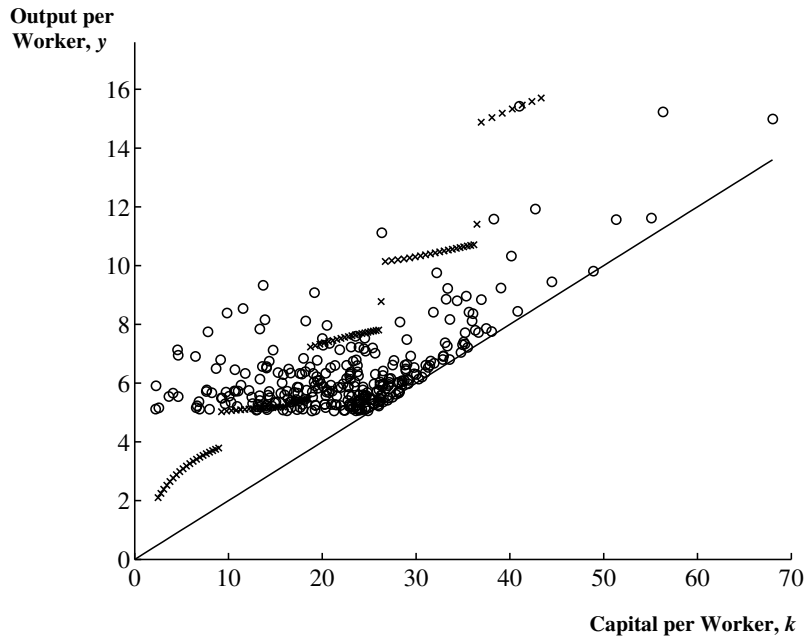

Note: Circles indicate ideas, plus signs indicate capital-output combinations that are actually used. The model is simulated for 100 periods with $N_{0}=50, \alpha=5$, $\beta=2.5, g=.10, \gamma_{a}=1, \gamma_{b}=0.2, k_{0}=2.5, s=0.2, \delta=.05$, and $\rho=-1$.

average growth rate of output per worker in this particular simulation is 1.48 percent, as compared to the theoretical value of 2 percent implied by the parameter values, given by $g / \alpha{ }^{25}$

Figure 6 plots the capital share $F_{K} K / Y$ over time. Even though the economy grows at a stable average rate, the capital share exhibits fairly large movements. When the economy is using a single production technique, the accumulation of capital leads the capital share to decline. Alternatively, when the economy is using two techniques to convexify the production set, the marginal product of capital is constant, so the capital share rises smoothly.

\footnotetext{
${ }^{25}$ We compute the average growth rate by dropping the first 20 observations (to minimize the effect of initial conditions) and then regressing the log of output per worker on a constant and a time trend.
} 


\section{FIGURE 5. Output per Worker over Time}

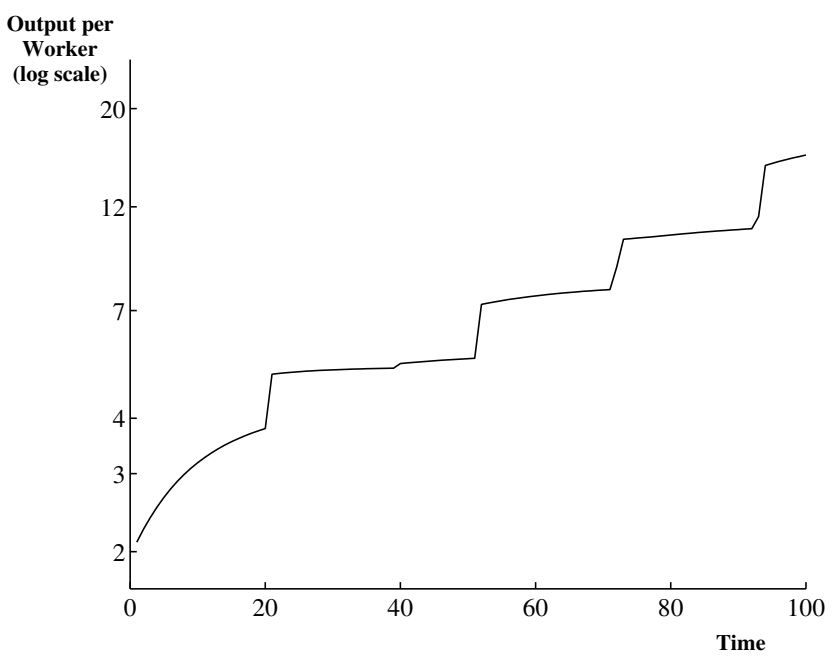

Note: See notes to Figure 4.

It is interesting to compare the behavior of the capital share in the Pareto model with the behavior that occurs in the simple baseline model. In the simple model, the economy equates the capital share to a function of the elasticity of the technology menu. If this elasticity is constant, then the capital share would be constant over time. Here, the technology menu exhibits a constant elasticity on average, but the menu is not a smooth, continuous function. Quite the opposite: the extreme value nature of this problem means the frontier is sparse, as the example back in Figure 1 suggests. This means the capital share will be stationary, but that it can move around, both as the economy accumulates capital and as it switches techniques.

Figure 7 shows the technology choices that occur in this simulation. As in Figure 4, the 300 ideas with the highest level of $y_{i}=a_{i}$ are plotted. This time, however, the $\left(a_{i}, b_{i}\right)$ pair corresponding to each idea is plotted. The 
FIGURE 6. The Capital Share over Time

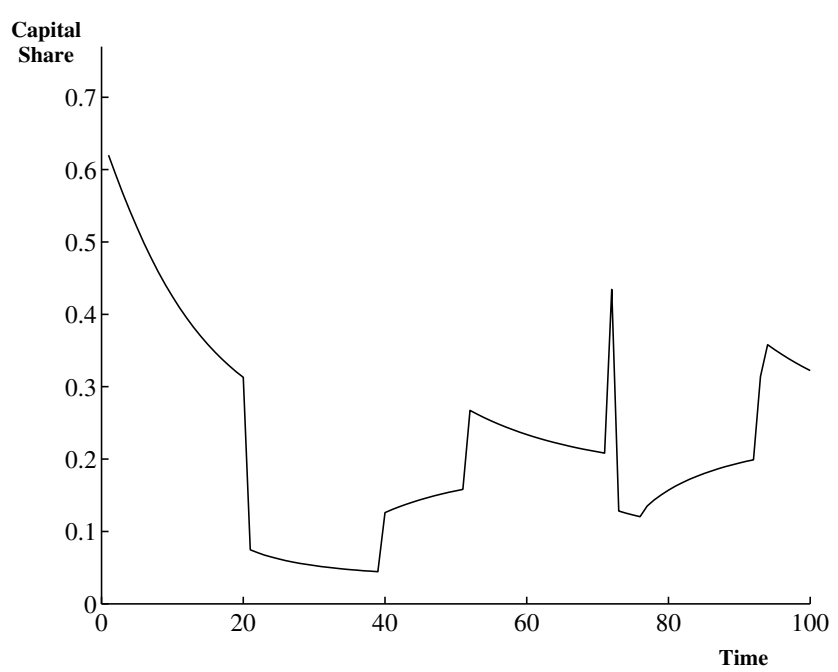

Note: See notes to Figure 4.

graph therefore shows the stochastic version of the technology menu. In addition, the figure plots with a "+" the idea combinations that are actually used as the economy grows over time. Corresponding to the theoretical finding earlier, one sees that the level of $b_{i}^{*}$ appears stationary, while the level of $a_{i}^{*}$ trends upward. On average, technological change is labor augmenting.

\section{CONCLUSION}

This paper provides microfoundations for the standard production function that serves as a building block for many economic models. An idea is a set of instructions that tells how to produce with a given collection of inputs. It can be used with a different mix of inputs, but it is not especially effective with the different mix; the elasticity of substitution in production is low for a given production technique. Instead, producing with a different input mix typically leads the production unit to switch to a new technique. 
FIGURE 7. Technology Choices

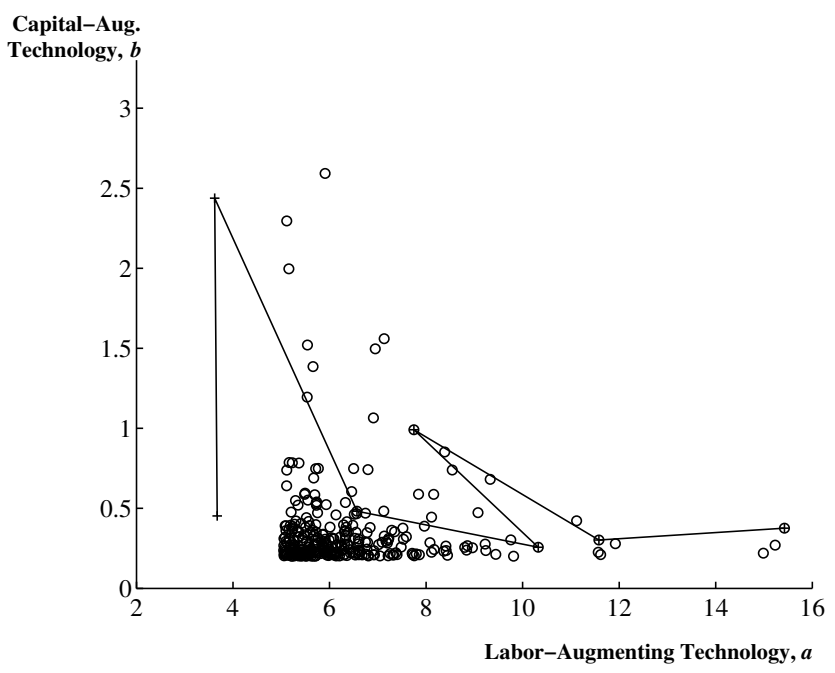

Note: From more than 1 million ideas generated, the 300 with the highest level of $a$ are plotted as circles. The figure also plots with a "+" the $\left(a_{i}, b_{i}\right)$ combinations that are used at each date and links them with a line. When two ideas are used simultaneously, the idea with the higher level of output is plotted. See also notes to Figure 4. 
This suggests that the shape of the global production function hinges on the distribution of available techniques.

Kortum (1997) examined a model in which productivity levels are draws from a distribution and showed that only distributions in which the upper tail is a power function are consistent with exponential growth. If one wants a model in which steady-state growth occurs, then one needs to build in a Pareto distribution for ideas. What we show here is that this assumption delivers two additional results. Pareto distributions lead the global production function to take a Cobb-Douglas form and produce a setup where technological change, in the long run, is entirely labor augmenting.

There are several additional directions for research suggested by this approach. First, our standard ways of introducing skilled and unskilled labor into production involve production functions with an elasticity of substitution bigger than one, consistent with the observation that unskilled labor's share of income seems to be falling. ${ }^{26}$ How can this view be reconciled with the reasoning here?

Second, the large declines in the prices of durable investment goods are often interpreted as investment-specific technological change. That is, they are thought of as increases in $b$ rather than increases in $a .^{27}$ This is the case in Greenwood, Hercowitz and Krusell (1997) and Whelan (2001), and it is also implicitly the way the hedonic pricing of computers works in the National Income and Product Accounts: better computers are interpreted as more computers. The model in this paper suggests instead that $b$ might be stationary, so there is a tension with this other work. Of course, it is not at all obvious that better computers are equivalent to more computers.

\footnotetext{
${ }^{26}$ See Katz and Murphy (1992) and Krusell, Ohanian, Rios-Rull and Violante (2000), for example.

${ }^{27}$ This is loose. In fact, they are thought of as increases in a term that multiplies investment in the capital accumulation equation. Of course, for many purposes this is like an increase in $b$.
} 
Perhaps a better computer is like having two people working with a single computer (as in extreme programming). In this case, better computers might be thought of as increases in $a$ instead. This remains an open question. Alternatively, it might be desirable to have microfoundations for a CobbDouglas production function that permits capital-augmenting technological change to occur in the steady state.

Finally, one might ask how the model relates to recent discussions about the behavior of capital shares. The literature is in something of a flux. For a long time, of course, the stylized fact has been that capital's share is relatively stable. This turns out to be true at the aggregate level for the United States and Great Britain, but it is not true at the disaggregated level in the U.S. or in the aggregate for many other countries. Rather, the more accurate version of the fact appears to be that capital's share can exhibit large medium term movements and even trends over periods longer than 20 years in some countries and industries. ${ }^{28}$ This paper is somewhat agnostic about factor shares. As shown in Figure 6, the Pareto model predicts the capital share may vary over time, while of course the baseline model implied a constant capital share. However, there are many other determinants of capital shares left out of this model, including aggregation issues and wedges between marginal products and prices, so care should be taken in interpreting the model along this particular dimension.

\footnotetext{
${ }^{28}$ The recent papers by Blanchard (1997), Bentolila and Saint-Paul (2003), and Harrison (2003) discuss in detail the facts about capital and labor shares and how they vary. Gollin (2002) is also related; that paper argues the in the cross-section of countries, labor shares are more similar than rough data on employee compensation as a share of GDP suggests because of the very high levels of self-employment in many poor countries.
} 


\section{APPENDIX: AN ALTERNATIVE DERIVATION OF THE COBB-DOUGLAS RESULT}

Here we drop the assumption that ideas arrive as a Poisson process and show that one still recovers the Cobb-Douglas result (and therefore the labor-augmenting technical change). The difference is that the result now holds asymptotically, as the number of ideas goes to infinity, and the proof involves the use of extreme value theory.

Let $N$ now denote the total number of ideas that have been discovered and drop the Poisson process. This is the only change to the model in Section 3. As before, let $Y_{i}$ denote production using technique $i$ with a given amount of capital and labor. Then

$$
\begin{aligned}
H(\tilde{y}) \equiv \operatorname{Prob}\left[Y_{i}>\tilde{y}\right] & =\operatorname{Prob}\left[b_{i} K>\tilde{y}, a_{i} L>\tilde{y}\right] \\
& =G\left(\frac{\tilde{y}}{K}, \frac{\tilde{y}}{L}\right) \\
& =\gamma K^{\beta} L^{\alpha} \tilde{y}^{-(\alpha+\beta)} .
\end{aligned}
$$

That is, the distribution of $Y_{i}$ is Pareto. ${ }^{1}$

The global production function is the maximum amount of output that can be produced with a single technique. Formally, it is defined as

$$
Y=F(K, L, N)=\max _{i=1, \ldots, N} Y_{i}
$$

Since the $N$ draws are independent,

$$
\begin{aligned}
\operatorname{Prob}[Y \leq \tilde{y}] & =(1-H(\tilde{y}))^{N} . \\
& =\left(1-\gamma K^{\beta} L^{\alpha} \tilde{y}^{-(\alpha+\beta)}\right)^{N} .
\end{aligned}
$$

Of course, as the number of ideas $N$ gets large, this probability for any given level of $\tilde{y}$ goes to zero. So to get a stable distribution, we need to normalize our random variable somehow, in a manner analogous to that used in the Central Limit Theorem.

\footnotetext{
${ }^{1}$ Since $b_{i} \geq \gamma_{b}$ and $a_{i} \geq \gamma_{a}$, the support for this distribution is $\tilde{y} \geq \max \left\{\gamma_{b} K, \gamma_{a} L\right\}$.
} 
In this case, the right normalization turns out to involve $z_{N}$, where

$$
z_{N} \equiv\left(\gamma N K^{\beta} L^{\alpha}\right)^{\frac{1}{\alpha+\beta}}
$$

In particular, consider

$$
\begin{aligned}
\operatorname{Prob}\left[Y \leq z_{N} \tilde{y}\right] & =\left(1-\gamma K^{\beta} L^{\alpha}\left(z_{N} \tilde{y}\right)^{-(\alpha+\beta)}\right)^{N} \\
& =\left(1-\frac{\tilde{y}^{-(\alpha+\beta)}}{N}\right)^{N} .
\end{aligned}
$$

Then using the standard result that $\lim _{N \rightarrow \infty}(1-x / N)^{N}=\exp (-x)$ for any fixed value of $x$, we have

$$
\lim _{N \rightarrow \infty} \operatorname{Prob}\left[Y \leq z_{N} \tilde{y}\right]=\exp \left(-\tilde{y}^{-(\alpha+\beta)}\right)
$$

for $\tilde{y}>0 .^{2}$

Therefore

$$
\frac{Y}{\left(\gamma N K^{\beta} L^{\alpha}\right)^{1 / \alpha+\beta}} \stackrel{a}{\sim} \text { Fréchet }(\alpha+\beta) .
$$

The global production function, appropriately normalized, converges asymptotically to a Fréchet distribution. We can think of the production function, asymptotically, as

$$
Y \approx\left(\gamma N K^{\beta} L^{\alpha}\right)^{\frac{1}{\alpha+\beta}} \epsilon
$$

where $\epsilon$ is a random variable drawn from a Fréchet distribution with parameter $\alpha+\beta$. This result can be compared to that in equation (25): we get the same result, asymptotically, that we obtained when we used the Poisson process for research.

\footnotetext{
${ }^{2}$ This is a special case of the much more general theory of extreme values. For a more general theorem relevant to this case, see Theorem 2.1.1 of Galambos (1978).
} 


\section{REFERENCES}

Acemoglu, Daron, "Factor Prices and Technical Change: From Induced Innovation to Recent Debates," in P. Aghion, R. Frydman, J. Stiglitz, and M. Woodford, eds., Knowledge, Information, and Expectations in Modern Macroeconomics, Princeton, N.J.: Princeton University Press, 2003.

__ "Labor- and Capital-Augmenting Technical Change," Journal of European Economic Association, March 2003, 1, 1-37.

Barro, Robert J. and Xavier Sala-i-Martin, Economic Growth, McGraw-Hill, 1995.

Basu, Susanto and David N. Weil, "Appropriate Technology and Growth," Quarterly Journal of Economics, November 1998, 113 (4), 1025-1054.

Bentolila, Samuel and Gilles Saint-Paul, "Explaining Movements in the Labor Share," 2003. CEMFI mimeo.

Bertran, Fernando Leiva, "Pricing Patents through Citations," November 2003. University of Rochester mimeo.

Blanchard, Olivier J., "The Medium Run," Brookings Papers on Economic Activity, 1997, 1997 (2), 89-141.

Caballero, Ricardo J. and Mohamad L. Hammour, "Jobless Growth: Appropriability, Factor Substitution, and Unemployment," Carnegie Rochester Conference Series on Public Policy, 1998, 48, 51-94.

Castillo, Enrique, Extreme Value Theory in Engineering, London: Academic Press, 1988.

Chevalier, Judith and Austan Goolsbee, "Price Competition Online: Amazon Versus Barnes And Noble," Quantitative Marketing and Economics, forthcoming 2004.

Cordoba, Juan Carlos, "On the Distribution of City Sizes," 2003. Rice University mimeo.

Cox, Raymond and Kee H. Chung, "A Stochastic Model of Superstardom: an application of the Yule distribution," Review of Economics and Statistics, November 1991, 73 (4), 740-747.

Drandakis, E.M. and Edmund S. Phelps, "A Model of Induced Invention, Growth, and Distribution," Economic Journal, December 1966, 76 (304), 823-840.

Gabaix, Xavier, “Zipf's Law for Cities: An Explanation," Quarterly Journal of Economics, August 1999, 114 (3), 739-767.

Galambos, Janos, The Asymptotic Theory of Extreme Order Statistics, New York: John Wiley \& Sons, 1978. 
Gilchrist, Simon and John C. Williams, "Putty Clay and Investment: A Business Cycle Analysis," Journal of Political Economy, October 2000, 108 (5), 928960.

Gollin, Douglas, "Getting Income Shares Right," Journal of Political Economy, April 2002, 110 (2), 458-474.

Grabowski, Henry, "Patents and New Product Development in the Pharmaceutical and Biotechnology Industries,” July 2002. Duke University mimeo.

Greenwood, Jeremy, Zvi Hercowitz, and Per Krusell, "Long-Run Implications of Investment-Specific Technological Change," American Economic Review, June 1997, 87 (3), 342-362.

Harhoff, Dietmar, Frederic M. Scherer, and Katrin Vopel, "Exploring the Tail of Patented Invention Value Distributions," 1997. WZB Working Paper 97-27.

Harrison, Ann E., "Has Globalization Eroded Labor's Share? Some Cross-Country Evidence," May 2003. U.C. Berkeley mimeo.

Houthakker, Henrik S., "The Pareto Distribution and the Cobb-Douglas Production Function in Activity Analysis," Review of Economic Studies, 1955-1956, 23 (1), 27-31.

Huber, John C., "Cumulative Advantage and Success-Breeds-Sucess: the Value of Time Pattern Analysis," Journal of the American Society for Information Science, 1998, 49 (5), 471-476.

Jones, Charles I., "R\&D-Based Models of Economic Growth,” Journal of Political Economy, August 1995, 103 (4), 759-784.

Katz, Lawrence and Kevin Murphy, "Changes in Relative Wages, 1963-1987: Supply and Demand Factors," Quarterly Journal of Economics, February 1992, 107 (1), 35-78.

Kennedy, Charles M., "Induced Bias in Innovation and the Theory of Distribution," Economic Journal, September 1964, 74 (295), 541-547.

Kortum, Samuel S., "Research, Patenting, and Technological Change," Econometrica, 1997, 65 (6), 1389-1419.

Krusell, Per, Lee Ohanian, Jose-Victor Rios-Rull, and Giovanni Violante, "CapitalSkill Complementarity and Inequality: A Macroeconomic Analysis," Econometrica, September 2000, 68 (5), 1029-1053.

Lagos, Ricardo, “A Model of TFP,” January 2004. NYU working paper.

Lotka, A.J., "The Frequency Distribution of Scientific Productivity," Journal of the Washington Academy of Sciences, 1926, 16, 317-323. 
Mitzenmacher, Michael, "A Brief History of Generative Models for Power Law and Lognormal Distributions," Internet Mathematics, forthcoming 2003, 1 (2).

Rossi-Hansberg, Esteban and Mark L. J. Wright, "Urban Structure and Growth," 2004. Stanford University mimeo.

Saez, Emmanuel, "Using Elasticities to Derive Optimal Tax Rates," Review of Economic Studies, 2001, 68, 205-229.

Samuelson, Paul A., "A Theory of Induced Innovations Along Kennedy-Weisacker Lines," Review of Economics and Statistics, 1965, 47, 343-356.

Sornette, Didier and Daniel Zajdenweber, "The economic return of research: the Pareto law and its implications," European Physical Journal B, 1999, 8 (4), 653-664. http://xxx.lanl.gov/abs/cond-mat/9809366.

_ and _ _ "Economy of Scales in R\&D with Block-busters," January 2000. UCLA mimeo.

Whelan, Karl, "Balance Growth Revisited: A Two-Sector Model of Economic Growth," 2001. Federal Reserve Board of Governors mimeo. 